\title{
Beyond Collins and Sivers: Further Measurements of the Target Transverse Spin-Dependent Azimuthal Asymmetries in Semi-Inclusive DIS from COMPASS
}

\author{
Aram Kotzinian * \\ on behalf of the COMPASS collaboration \\ INFN, Sezione di Torino, Via P. Giuria 1, I-10125 Torino, Italy
}

\begin{abstract}
In semi-inclusive DIS of polarized leptons on a transversely polarized target eight azimuthal modulations appear in the cross-section. Within QCD parton model four azimuthal asymmetries can be interpreted at leading order, two of them being the already measured Collins and Sivers asymmetries. The other two leading twist asymmetries, related to different transverse momentum dependent quark distribution functions, and also additional four asymmetries which can be interpreted as twist-three contributions have been measured for the first time at COMPASS, using a $160 \mathrm{GeV} / \mathrm{c}$ longitudinally polarized $\left(P_{\text {beam }} \simeq-0.8\right)$ muon beam and a transversely polarized ${ }^{6} \mathrm{LiD}$ target. We present here the preliminary results from the 2002-2004 data.
\end{abstract}

\section{Introduction}

During last few years many exciting experimental results and theory development are obtained in SIDIS on the transversely polarized target. Up to now only the measurements $[2,3$, 4] of Sivers and Collins asymmetries were performed by HERMES and COMPASS collaborations and together with data from BELLE [5] they allow a first extraction the transversity and Sivers transverse momentum dependent (TMD) distribution functions (DFs) and Collins fragmentation function (FF). In addition to these, the general expression of SIDIS cross section [6] contains six more target transverse polarization dependent azimuthal asymmetries. Here we present the preliminary results on these asymmetries for the first time measured by COMPASS from the 2002-2004 data.

\subsection{Definition of asymmetries}

In the following the notations of Ref. [7] are used. There are eight azimuthal modulations related to the target transverse polarization:

$$
\begin{aligned}
& w_{1}\left(\phi_{h}, \phi_{s}\right)=\sin \left(\phi_{h}-\phi_{s}\right), w_{2}\left(\phi_{h}, \phi_{s}\right)=\sin \left(\phi_{h}+\phi_{s}\right), w_{3}\left(\phi_{h}, \phi_{s}\right)=\sin \left(3 \phi_{h}-\phi_{s}\right), \\
& w_{4}\left(\phi_{h}, \phi_{s}\right)=\sin \left(\phi_{s}\right), w_{5}\left(\phi_{h}, \phi_{s}\right)=\sin \left(2 \phi_{h}-\phi_{s}\right), w_{6}\left(\phi_{h}, \phi_{s}\right)=\cos \left(\phi_{h}-\phi_{s}\right), \\
& w_{7}\left(\phi_{h}, \phi_{s}\right)=\cos \left(\phi_{s}\right), w_{8}\left(\phi_{h}, \phi_{s}\right)=\cos \left(2 \phi_{h}-\phi_{s}\right),
\end{aligned}
$$

*On leave from Yerevan Physics Institute, 375036 Yerevan, Armenia and JINR, 141980 Dubna, Russia. 
where first two correspond to Sivers and Collins effects. The expression for the cross section in interest can be represented as

$$
\begin{aligned}
d \sigma\left(\phi_{h}, \phi_{s}, \ldots\right) \propto & \propto\left(1+\left|\mathbf{S}_{T}\right| \sum_{i=1}^{5} D^{w_{i}\left(\phi_{h}, \phi_{s}\right)} A_{U T}^{w_{i}\left(\phi_{h}, \phi_{s}\right)} w_{i}\left(\phi_{h}, \phi_{s}\right)\right. \\
& \left.+\quad P_{\text {beam }}\left|\mathbf{S}_{T}\right| \sum_{i=6}^{8} D^{w_{i}\left(\phi_{h}, \phi_{s}\right)} A_{L T}^{w_{i}\left(\phi_{h}, \phi_{s}\right)} w_{i}\left(\phi_{h}, \phi_{s}\right)+\ldots\right)
\end{aligned}
$$

where $\mathbf{S}_{T}$ is the target transverse polarization. We factored out the explicitly calculable depolarization factors, $D^{w_{i}\left(\phi_{h}, \phi_{s}\right)}$, and defined the asymmetries as the ratios of corresponding structure functions to unpolarized one:

$$
A_{B T}^{w_{i}\left(\phi_{h}, \phi_{s}\right)} \equiv \frac{F_{B T}^{w_{i}\left(\phi_{h}, \phi_{s}\right)}}{F_{U U, T}}
$$

where $B=L$ or $B=U$ corresponds to beam polarization dependent or independent part of asymmetry.

The depolarization factors entering in Eq. (2) depend only on $y$ and are given as

$$
\begin{aligned}
& D^{\sin \left(\phi_{h}-\phi_{s}\right)}(y)=1, \quad D^{\cos \left(\phi_{h}-\phi_{s}\right)}(y)=\frac{y(2-y)}{1+(1-y)^{2}}, \\
& D^{\sin \left(\phi_{h}+\phi_{s}\right)}(y)=D^{\sin \left(3 \phi_{h}+\phi_{s}\right)}(y)=\frac{2(1-y)}{1+(1-y)^{2}}, \\
& D^{\sin \left(2 \phi_{h}-\phi_{s}\right)}(y)=D^{\sin \left(\phi_{s}\right)}(y)=\frac{2(2-y) \sqrt{1-y}}{1+(1-y)^{2}} \\
& D^{\cos \left(2 \phi_{h}-\phi_{s}\right)}(y)=D^{\cos \left(\phi_{s}\right)}(y)=\frac{2 y \sqrt{1-y}}{1+(1-y)^{2}} .
\end{aligned}
$$

The asymmetries extracted from the data as amplitudes of corresponding azimuthal modulations (raw asymmetries) are then given by

$$
\begin{aligned}
& A_{U T, \text { raw }}^{w_{i}\left(\phi_{h}, \phi_{s}\right)}=D^{w_{i}\left(\phi_{h}, \phi_{s}\right)}(y) f\left|S_{T}\right| A_{U T}^{w_{i}\left(\phi_{h}, \phi_{s}\right)}, \quad(i=1,5), \\
& A_{L T, \text { raw }}^{w_{i}\left(\phi_{h}, \phi_{s}\right)}=D^{w_{i}\left(\phi_{h}, \phi_{s}\right)}(y) f P_{\text {beam }}\left|S_{T}\right| A_{L T}^{w_{i}\left(\phi_{h}, \phi_{s}\right)}, \quad(i=6,8),
\end{aligned}
$$

where $f$ is the target polarization dilution factor.

In the QCD parton model the asymmetries $A_{L T}^{\cos \left(\phi_{h}-\phi_{s}\right)}$ and $A_{U T}^{\sin \left(3 \phi_{h}-\phi_{s}\right)}$ are given by the ratio of convolutions of spin-dependent to spin-independent twist two DFs and FFs, for example

$$
A_{U T}^{\sin \left(3 \phi_{h}-\phi_{s}\right)}=\frac{h_{1 T}^{\perp q} \otimes H_{1 q}^{\perp h}}{f_{1}^{q} \otimes D_{1 q}^{h}}
$$

and can be used for extraction of DFs $g_{1 T}^{q}$ and $h_{1 T}^{\perp q}$ describing the quark longitudinal and transverse (along quark transverse momentum) polarization in the transversely polarized nucleon. The other asymmetries can be interpreted as Cahn kinematic corrections to spin effects on the transversely polarized nucleon [6], for example:

$$
A_{L T}^{\cos \left(\phi_{s}\right)}=\frac{M}{Q} \frac{g_{1 T}^{q} \otimes D_{1 q}^{h}}{f_{1}^{q} \otimes D_{1 q}^{h}}
$$



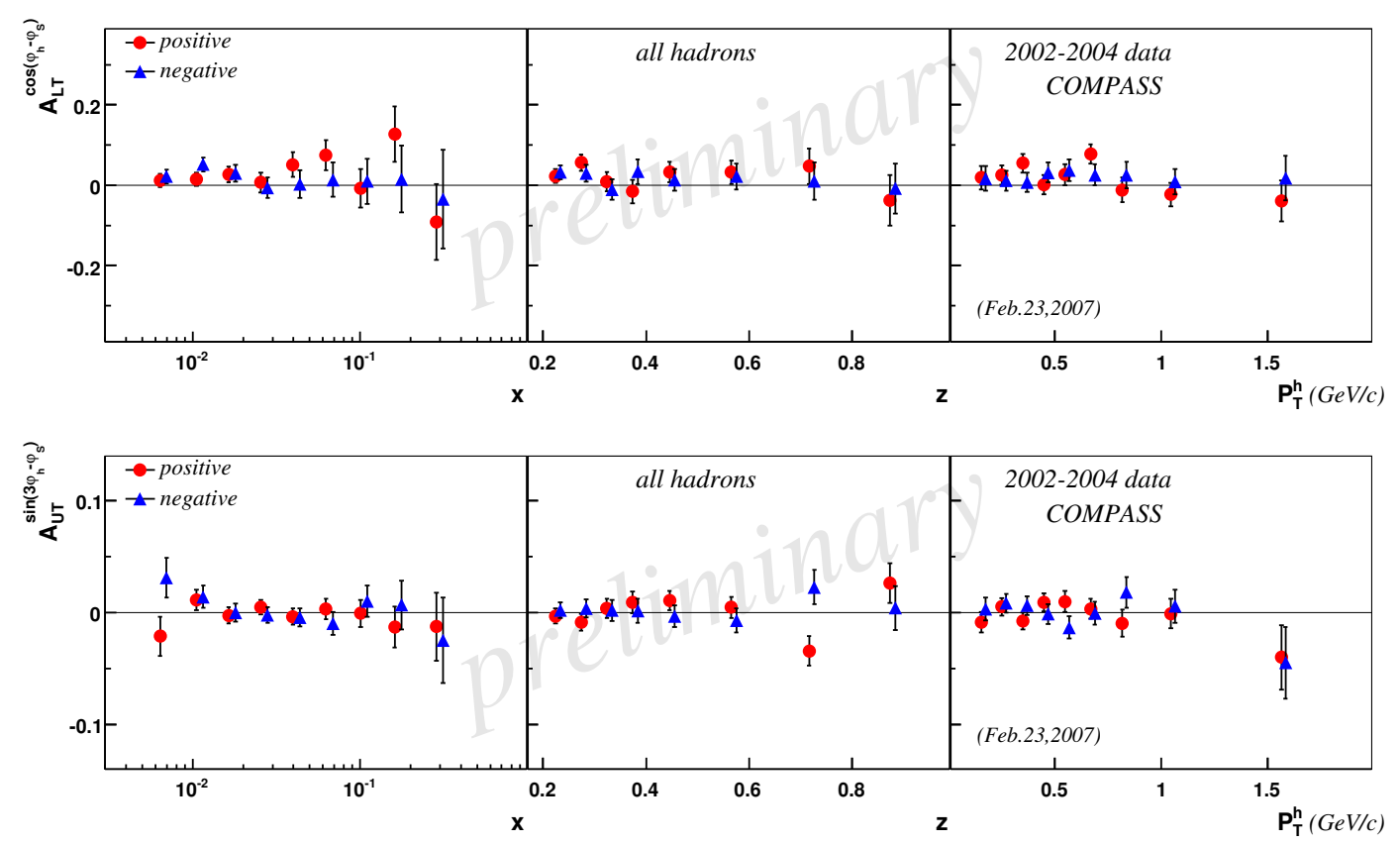

Figure 1: The asymmetries $A_{L T}^{\cos \left(\phi_{h}-\phi_{s}\right)}$ and $A_{U T}^{\sin \left(3 \phi_{h}-\phi_{s}\right)}$ as a function of $x, z$ and $P_{T}^{h}$.

\section{Results}

The event selection and asymmetry extraction are done as described in [4]. The following kinematic cuts were imposed: $Q^{2}>1(\mathrm{GeV} / \mathrm{c})^{2}, W>5 \mathrm{GeV}, 0.1<y<0.9, P_{T}^{h}>0.1$ $\mathrm{GeV} / \mathrm{c}$ and $z>0.2$. In Figs. 1 and 2 for the first time we present six target transverse spin dependent asymmetries extracted from COMPASS 2002-2004 data collected on deuterium target. The estimated systematic errors are smaller than statistical. All six newly measured asymmetries are compatible with zero within statistical errors.

\section{References}

[1] Slides: http: //indico. cern. ch/contributionDisplay. py? contribId=168\&sessionId=4\&conf $I d=9499$

[2] A. Airapetian et al. [HERMES Collaboration], Phys. Rev. Lett. 94, 012002 (2005)

[3] V. Y. Alexakhin et al. [COMPASS Collaboration], Phys. Rev. Lett. 94, 202002 (2005) [arXiv:hepex/0503002].

[4] E. S. Ageev et al. [COMPASS Collaboration], Nucl. Phys. B 765, 31 (2007) [arXiv:hep-ex/0610068];

[5] R. Seidl et al. [Belle Collaboration], Phys. Rev. Lett. 96, 232002 (2006) [arXiv:hep-ex/0507063].

[6] A. Kotzinian, Nucl. Phys. B 441, 234 (1995) [arXiv:hep-ph/9412283].

[7] A. Bacchetta, M. Diehl, K. Goeke, A. Metz, P. Mulders and M. Schlegel, JHEP 0702, 093 (2007) [arXiv:hep-ph/0611265]. 

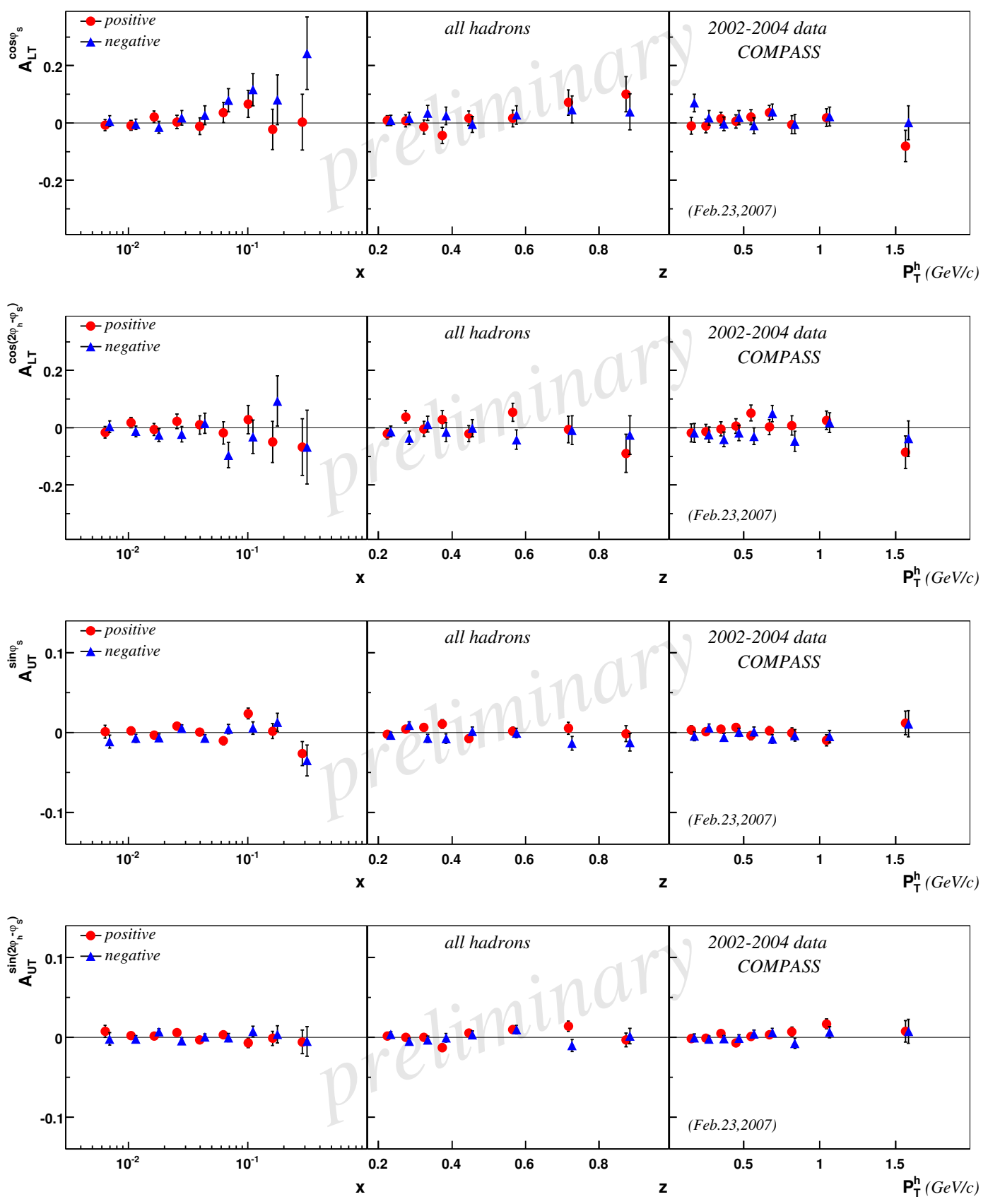

Figure 2: The asymmetries $A_{L T}^{\cos \phi_{s}}, A_{L T}^{\cos \left(2 \phi_{h}-\phi_{s}\right)}, A_{U T}^{\sin \phi_{s}}$ and $A_{U T}^{\sin \left(2 \phi_{h}-\phi_{s}\right)}$ as a function of $x$, $z$ and $P_{T}^{h}$. 